\title{
Haidt y Gigerenzer: la decisión moral como respuesta automática*
}

Haidt and Gigerenzer: moral decision and automatic response

Haidt y Gigerenzer: a decisão moral como resposta automática

Jonathan Echeverri Álvarez**

Recibido 28. 02. 2016 • Arbitrado 26. 03. 2016 • Aprobado 13.04. 2016

\section{Resumen}

Este artículo de revision tiene el objetivo de discutir dos modelos naturalistas contemporáneos que se aproximan a explicar la decisión moral como respuesta automática: la propuesta de Jonathan Haidt y la de Gerd Gigerenzer. Las propuestas explicativas de Haidt y Gigerenzer resaltan el papel de la intuición y la emoción en las decisiones morales. De tal forma que estos procesos automáticos son ubicuos en las decisiones cotidianas, constituyen un recurso para enfrentar la incertidumbre y sesgan la deliberación para tomar una decisión moral. Sin embargo, ambos autores restan importancia, en sus propuestas explicativas, al uso que puede tener la deliberación para cuestionar e incorporar nuevos automatismos en la decisión moral.

Palabras clave: decisión moral, intuición, razonamiento estratégico, racionalidad limitada.

Este artículo de investigación se deriva del proyecto de maestría titulado "Decisión moral como respuesta automática. Consideraciones sobre un debate naturalista" en el periodo 2013-2016. Maestría en Filosofía. Línea en Filosofía de la Ciencia. Instituto de Filosofía. Universidad de Antioquia.

** Psicólogo egresado de la Universidad de Antioquia. Magíster en Filosofía. Línea en Filosofia de la Ciencia. Instituto de Filosofía. Universidad de Antioquia. Integrante activo del Grupo de Investigación en Conocimiento, Filosofía, Ciencia, Historia y Sociedad y el Grupo Intersdiciplinario en Estudios Sociales GIES. Docente e investigador de la Corporación Universitaria Minuto de Dios, seccional Bello. Y profesor de cátedra de la Universidad EAFIT, jonathantyche@gmail.com. 


\section{Abstract}

This article aims to discuss two contemporary models that approximate naturalists explain the moral decision as an automatic response: Jonathan Haidt's proposal and Gerd Gigerenzer. Explanatory Haidt and Gigerenzer proposals highlight the role of intuition and emotion in moral decisions. So that these automatic processes are ubiquitous in everyday decisions are a resource to face the uncertainty and bias deliberation to make a moral decision. However, both authors downplay, in its explanatory proposals, the use that can have the deliberation to challenge and incorporate new automation in moral decision.

Key words: moral decision, insight, strategic thinking, bounded rationality.

\section{Resumo}

Este artigo tem o objetivo de discutir os modelos naturalistas contemporâneos que se aproximam a explicar a decisão moral como resposta automática: a proposta de Jonathan Haidt e a de Gerd Gigerenzer. As propostas explicativas de Haidt e Gigerenzer realçam o papel da intuição e da emoção nas decisões morais. De tal forma que estes processos são ubíquos nas decisões cotidianas, constituem um recurso para enfrentar a incerteza e rasgam a deliberação para tomar uma decisão moral. Porém, ambos autores subtraem importância, em suas propostas explicativas, ao uso que pode ter a deliberação para questionar e incorporar novos automatismos na decisão moral.

Palavras chave: decisão moral, intuição, raciocínio estratégico, racionalidade limitada. 


\section{Introducción}

Las ideas de Jonathan Haidt (2001, 2006, 2012) y Gerd Gigerenzer (2008b, 2008c, 2010) representan en la actualidad dos puntos de referencia teóricos que ilustran la incidencia del automatismo en el comportamiento moral. La propuesta explicativa de Haidt (2001, 2012), en una de sus tesis constitutivas, resalta el papel de la intuición y la emoción para evocar juicios y tomar decisiones morales. En principio, el trabajo de Haidt (2001) se publicó con el propósito de ofrecer una explicación sobre el juicio moral generado por una respuesta automática donde el razonamiento o la deliberación consciente tiene el papel de justificar este juicio y persuadir a otros del mismo, luego, tal consideración se aplica de forma similar para explicar la ejecución de una decisión moral como respuesta automática (Haidt, 2006, 2012; Haidt \& Bjorklund, 2008a). En este contexto, emitir un juicio moral consiste en ofrecer una apreciación de aprobación o censura de una acción. Y tomar una decisión moral, a grandes rasgos, implica optar por una opción de respuesta que incide en el bienestar de otras personas, en un contexto moral específico. En ambos casos, Haidt (2006, 2012) sugiere que la intuición cumple un papel de vital importancia previo a un ejercicio de razonamiento consciente. Por otro lado, la investigación de Gigerenzer (2008b, 2010) resalta la racionalidad limitada con la cual la especie humana toma decisiones morales. Es decir, el ser humano toma decisiones con restricción en el tiempo, una ignorancia parcial de las alternativas y ante la presencia de un futuro incierto. En estas condiciones la racionalidad humana utiliza intuiciones como atajos para escoger las alternativas razonablemente buenas para un individuo en concreto, sin evaluar con detenimiento todas las opciones de respuesta en un problema de elección moral.

Este artículo muestra una relación teórica entre ambas propuestas naturalistas para explicar la decisión moral como repuesta automática, reconociendo, además, que ambos modelos aún se encuentran en una álgida discusión al interior de la comunidad científica, en la ética naturalista. Este texto se compone de tres apartados. En el primer apar- 
tado, se presenta el modelo de Haidt y se hace énfasis en la siguiente tesis del autor: cuando enfrentamos una situación que demanda tomar una decisión moral, primero experimentamos una intuición moral (ocurrencia, corazonada o sentimiento) y posteriormente usamos la razón para justificar y persuadir a otras personas sobre decisiones morales previamente tomadas. En segundo lugar, se expone el modelo de Gigerenzer sobre la moral en racionalidad limitada. Según este autor, la conducta moral (como juicio, decisión y acción) funciona en medio de la ignorancia parcial, un tiempo limitado y un futuro incierto, a partir de intuiciones morales que permiten aprovechar un motivo que satisface, es decir, que es razonablemente bueno para decidir. En tercer lugar, se considera una explicación de la decisión moral como respuesta automática con las ideas presentes en los modelos naturalistas propuestos por ambos autores. En tal caso, se plantea que Haidt y Gigerenzer, además de señalar la importancia que tiene la intuición y la emoción moral para tomar decisiones en condiciones de incertidumbre, ambos restan importancia al uso que puede tener la deliberación consciente para cuestionar e incorporar nuevos automatismos en las decisiones morales.

\section{Intuición y diversidad moral}

Tres tesis componen el modelo explicativo sobre la moral propuesto por Jonathan Haidt. La primera tesis sugiere que los juicios y decisiones morales son generados por intuición. Ante una situación que exige un juicio o una decisión moral, los seres humanos experimentan primero una intuición o una emoción y luego usan la razón para justificar y persuadir a otros (Greene \& Haidt, 2002; Haidt, 2001, 2006, 2012; Haidt \& Bjorklund, 2008a, 2008b). La segunda tesis plantea que la especie humana dispone de un primer borrador biológico de la moral con rasgos naturales compartidos de forma universal que, a través de la experiencia y el aprendizaje cultural, adopta formas concretas en sistemas morales diversos. La herencia biológica en la especie brinda un borrador moral flexible, susceptible de ser modificado, y el aprendizaje 
cultural permite modificar y actualizar, a la manera de un editor, este primer recurso natural (Haidt, 2007, 2012; Haidt \& Joseph, 2008). La tercera tesis expone que la moral permite conformar grupos con individuos que cooperan pero al mismo tiempo ésta constituye un velo que enceguece, es decir, no permite percibir los motivos morales inconscientes que suscitan tensiones entre grupos con creencias diferentes (Haidt, 2006, 2012; Haidt \& Kesebir, 2010). La moral es un rasgo biológico y cultural que une a las personas, pero al mismo tiempo permite concebir a otros grupos con creencias distintas como un conjunto de individuos moralmente equivocados. Incluso, con frecuencia, éstos son percibidos como enemigos que necesitan ser corregidos; sus concepciones de lo bueno y lo malo requieren una corrección inmediata (Haidt, 2007, 2012).

Haidt (2012) plantea tres metáforas para referirse a las tesis constitutivas de su modelo explicativo. En relación con la primera tesis, el autor resalta que la mente está dividida: un jinete que intenta domar un elefante. El jinete hace referencia al razonamiento consciente (proceso y respuesta deliberada) y el elefante a la intuición en sus reacciones pasionales (proceso y respuesta automática). Sin embargo, el trabajo del jinete consiste en servir al elefante y no al contrario como supone a primera vista tal interacción. En función de la segunda tesis, a propósito de la segunda metáfora, Haidt (2012) expone que la mente moral en parte es como un órgano sensorial con seis receptores sensoriales distintos. El autor supone que el primer borrador de la moral, con el cual todos los seres humanos nacen, es similar a una lengua con seis papilas gustativas distintas. Estas seis papilas gustativas son las siguientes: cuidado/daño (preocupación por el sufrimiento de los demás con bondad y compasión); justicia/reciprocidad (concierne al trato injusto, el engaño y nociones más abstractas como la justicia); lealtad al grupo (preocupación relacionada con obligaciones al pertenecer a un grupo: lealtad, auto sacrificio por el grupo y vigilancia contra la traición); auto$\mathrm{ridad} / \mathrm{respeto}$ (hace referencia al orden social y a las obligaciones de las relaciones jerárquicas: obediencia, respeto y cumplimiento del deber); y pureza/santidad (preocupación relacionada con el contagio físico y 
espiritual: castidad, salubridad y control de deseos) (Haidt \& Kesebir, 2010). A partir de estos rasgos universales, la especie humana, con la cultura, construye sistemas morales diversos haciendo énfasis en uno o varios rasgos. De igual forma, estos rasgos son las fuentes básicas de las intuiciones morales. Finalmente, la metáfora central propuesta, de acuerdo a la tercera tesis, consiste en que los seres humanos son noventa por ciento chimpancés y diez por ciento abejas. Esta metáfora sirve para ilustrar el funcionamiento de la selección natural en la moral, en dos niveles simultáneos. Un nivel individual donde resalta el egoísmo y la competencia (noventa por ciento chimpancés) y un nivel grupal que permite la conformación de equipos con individuos que cooperan y son altruistas al interior de estos grupos (diez por ciento abejas). Estos dos niveles hacen de los seres humanos una especie que compite pero a la vez coopera. Lo cual, según Haidt (2012), facilita la existencia y tensión entre egoísmo y altruismo, y el surgimiento de fenómenos culturales que derivan de tales rasgos como el heroísmo, la guerra y el genocidio.

En este artículo interesa especialmente la primera tesis con su consecuente metáfora. Esto sin desconocer la importancia, en el modelo explicativo, de las otras dos tesis constitutivas, puesto que estas dos últimas ideas son el telón de fondo que sugiere una evolución de la moral en los seres humanos. De acuerdo con Haidt \& Joseph (2008), la moral es parcialmente natural o biológica y parcialmente convencional o cultural. Ésta existe como un fenómeno natural entre la universalidad de sus rasgos y la relatividad de los sistemas morales que se crean a partir del primer borrador ofrecido por la naturaleza. De ahí la importancia notable de las otras dos tesis constitutivas del modelo explicativo en relación con el juicio y la decisión moral que surgen de forma automática.

A propósito de la primera tesis, Haidt $(2001,2012)$ plantea que por definición el juicio y la decisión moral son productos de un proceso automático (hace referencia a la intuición) y el proceso deliberado (razonamiento consciente) se experimenta como un suceso psicológico posterior. El razonamiento consciente sirve para justificar un juicio o una decisión ya tomada y persuadir a otros para que asuman un juicio 
o una decisión similar. Lo que está aquí en discusión es la motivación psicológica de una decisión moral: ¿̇Intuición o razón? ¿Ambas tal vez? ¿De qué forma?. La explicación ofrecida por el autor pone a la intuición en un primer plano mientras que la razón adquiere un papel instrumental secundario de justificación y persuasión. Contrario a la postura que asume el uso de la razón como un momento previo y fundamental para suscitar un juicio o decisión moral (Chang, 2009, 2012; Koorsgard, 2004, 2007).

$\mathrm{Al}$ respecto, es necesario distinguir entre la descripción y la prescripción moral. Una cosa es procurar describir y explicar el comportamiento moral; por ejemplo, enfocarse en identificar los mecanismos psicológicos que posibilitan la expresión de la moral en juicios o decisiones. En este escenario se inscribe el modelo explicativo de Haidt (2001, 2012). Y otra cosa bien distinta es prescribir la forma como los seres humanos deben comportarse. El primer proyecto, con énfasis en la descripción y explicación, es propio de la psicología moral, en el contexto más amplio de la ética naturalista; y el segundo proyecto, de la prescripción, pertenece a la filosofía moral (Antony, 2000; Babbitt, 2000; Camps, 2013; Cela, 1999; Tiberius, 2015). Esta distinción es importante en tanto que tradicionalmente los filósofos han propuesto que la razón debe constituir la principal motivación psicológica en todo juicio, decisión y acción moral (Camps, 2013; Chang, 2009; Koorsgard, 2004, 2007; Gigerenzer, 2008a; Haidt, 2001, 2012; Tiberius, 2015); sin embargo, la psicología ha constatado que, en la vida cotidiana, los juicios, decisiones y acciones morales no están motivadas principalmente por la razón, sino que hay otros factores que intervienen tales como la intuición y la emoción (Damasio, 1999, 2007; De Waal, 2007; Gigerenzer, 2008b, 2008c, 2010; Haidt, 2001, 2007, 2012; Sadler-Smith, 2008; Sunstein, 2005; Greene \& Haidt, 2002). Por lo tanto, para explicar las decisiones, acciones y juicios morales reales del día a día es necesario tener en cuenta estos otros factores psicológicos.

¿Cuáles son las razones prácticas que deben preceder la ejecución de una decisión moral?. Obtener una respuesta ha configurado parte 
de varias propuestas filosóficas en la historia de la ética (Camps, 2013). En cambio, ¿̇cuáles son los procesos psicológicos que anteceden la ejecución de una decisión moral?.Esta es una inquietud habitual en las discusiones de la ética naturalista. La tesis de Haidt (2001, 2012) es que no hay algo así como una razón o varias razones prácticas que sean previas a una decisión moral, sino que, lo que motiva la acción, es una intuición que se experimenta de dos formas distintas: una ocurrencia inmediata o una sensación indescriptible (presentimiento que no se puede explicar); o también una emoción moral (compasión, reciprocidad, empatía, amor o incluso odio, egoísmo, deseo de venganza) (Damasio, 2007; De Waal, 2007; Gigerenzer, 2008b, 2008c, 2010; Haidt, 2001; Nussbaum, 1995, 2008; Prinz, 2007; Prinz \& Nichols, 2010). Posteriormente, justificamos con la razón estas ocurrencias, presentimientos o emociones morales. En otras palabras, el ser humano puede pensar que delibera de forma consciente antes de tomar una decisión pero realmente hay un sesgo previo generado por la intuición o la emoción, una preferencia de la cual no somos del todo conscientes ni siquiera cuando deliberamos.

Consideremos un ejemplo. En un contexto político y social cercano en cualquier momento podemos estar exigidos a proferir un juicio o tomar una decisión a favor o en contra de la adopción homoparental; es decir, de la adopción de niños y niñas por parte de parejas homosexuales. Una vez que anunciamos un juicio de aprobación o censura moral, o tomamos una decisión al respecto, hacemos uso de la razón para justificar dicho juicio o decisión, y a la vez intentamos persuadir a otros de estas reflexiones deliberadas. Sin embargo, el modelo explicativo de Haidt $(2001,2012)$ permite inferir que posiblemente haya una intuición moral arraigada en una creencia religiosa o política (conservadora o liberal), que intentamos justificar a toda costa, aun si esto implica ofrecer interpretaciones tergiversadas de la evidencia empírica, proveniente de la ciencia, a nuestro favor. Esta forma de proceder aplica en otros temas morales de relevancia política y social: la eutanasia, el aborto, la legalización de la marihuana, entre otros. Y en la vida cotidiana, la especie humana enfrenta continuamente proble- 
mas de elección moral que implican ayuda, cooperación o daño a otras personas: colaborar con alguien en algún trabajo académico; ayudar a algún desconocido invidente a cruzar una calle; permitir o no a una hija en temprana edad jugar con "juguetes de hombres" o viceversa (permitir a los niños jugar con "juguetes de mujeres"); golpear a un niño o una niña como castigo por una "mala conducta" o causar daño físico al infante con el fin de posibilitar supuestamente un mejor aprendizaje; entre otra gran cantidad de casos.

Por otra parte, es complejo establecer una distinción entre decisiones amorales y decisiones morales, y esto porque los seres humanos en su complejidad social toman decisiones que continuamente afectan a otras personas. Desde esta perspectiva, las decisiones morales, aquellas que inciden en el bienestar de otros a través de la cooperación o el altruismo, son bastante frecuentes. Desde la perspectiva del funcionamiento del cerebro, tomamos decisiones automáticas o deliberadas; desde la perspectiva del contexto cultural que habitamos, tomamos decisiones automáticas o deliberadas pero que tienen un carácter económico, político o moral de acuerdo a la situación que exige estas elecciones. Las decisiones adquieren un carácter moral cuando se pone en juego el deseo, la necesidad o el bienestar del otro (Bartels, 2008; Bartels et al., 2015; Churchland, 2006; Churchland \& Suhler, 2014; Illiev et.al., 2009).

La propuesta explicativa de Haidt $(2001,2012)$ sugiere una reivindicación del sentimentalismo moral presente en la obra de Hume (2014) y Smith (2012). Se trata de un modelo naturalista que ofrece evidencia empírica a favor de la tesis principal propuesta por Hume (2014) de la primacía de la pasión sobre la razón en la motivación del juicio moral, y a favor de la tesis de Smith (2012) en relación con la simpatía o "el sentir con los otros" como el principal sentimiento moral que guía los juicios y las acciones morales. Haidt $(2001,2012)$ plantea que la primacía de la razón característica de la filosofía moral, en la reflexión prescriptiva, tuvo una incidencia directa en los modelos explicativos de la psicología moral durante las décadas de 1970 y 1980. Es decir, durante 
dos décadas la incipiente investigación sobre el juicio moral suponía que el razonamiento consciente es el principal recurso psicológico que utilizan las personas para guiar el comportamiento moral.

En este escenario, el infante adquiere madurez moral en la medida en que aprende a hacer uso de la razón con el fin de generar y argumentar juicios y decisiones morales. Sólo hasta la década de 1990 la ciencia prestó atención al papel de la intuición y la emoción en la conducta moral. La tensión tradicional entre racionalistas y sentimentalistas en filosofía moral, está presente en los modelos explicativos actuales de la moral; es decir, la misma tensión se sostiene en el contexto de la ética naturalista. Existen modelos explicativos racionalistas que dan primacía a la razón sobre el sentimiento en el juicio y la decisión moral sin desconocer del todo la incidencia causal del sentimiento (Dennett, 2004; Hershberger, 2014; Koorsgard, 2007; Narvaez, 2008; Tiberius, 2015; Tomasello, 2014; Turiel, 2006), y hay modelos explicativos sentimentalistas que enfatizan en la primacía del sentimiento sobre la razón en el juicio y la decisión moral (Damasio, 1999, 2007; De Waal, 2007; Gigerenzer, 2008a, 2008b; Gigerenzer \& Sturn, 2012; Greene, 2013; Greene \& Haidt, 2002; Haidt, 2001, 2006, 2007, 2012).

Haidt (2001, 2012) llama al modelo que da primacía al sentimiento o intuición: The Social Intuitionist Model (SIM). Este modelo expone cuatro enlaces que inician su funcionamiento a partir de un estímulo o situación que evoca un juicio moral. Y otros dos vínculos que para el autor ocurren con poca frecuencia. Una vez que la especie humana enfrenta una situación que exige un juicio de aprobación o censura moral, entran en marcha los siguientes cuatro fenómenos psicológicos vinculados entre sí: 1. Juicio intuitivo; 2. Razonamiento de justificación posterior al juicio; 3. Razonamiento persuasivo; y, finalmente, 4. La persuasión social. Los otros dos vínculos usados con poca frecuencia, son: 5. Juicio reflexivo por deliberación; y 6. Reflexión privada. Por ejemplo, frente al caso de adopción por parte de parejas homoparentales alguien emite inmediatamente un juicio de censura moral porque no considera tal adopción moralmente conveniente. Es decir, 1. Siente o intuye que 
tal adopción es algo malo y antinatural que no debe ser permitido. Luego, 2. Intenta justificar este juicio moral recurriendo a argumentos religiosos, políticos, sociales y, tal vez, científicos. Después, 3. Convierte esta justificación en un recurso persuasivo. Al final, 4. Intenta persuadir a los demás de la censura moral en contra de tal adopción. Con poca frecuencia, la persona antes de emitir un juicio apresurado de censura moral, 5. Se detiene a reflexionar en relación con todas las variables en juego a tener en cuenta en esta situación (consecuencias positivas y negativas para tal adopción en los niñas, niñas y las parejas que deseen adoptar; la evidencia científica al respecto disponible; experiencia de otros países donde es legal y socialmente permisible tal adopción; el contexto social particular donde se encuentra en discusión esta posibilidad moral de adopción; entre otros aspectos a tener en cuenta y que son relevantes); y 6. Habitualmente el individuo no reflexiona en torno a cómo sus propias creencias morales, convicciones religiosas y/o políticas y aprendizajes culturales previos, sesgan la emisión de un juicio moral que tiene efectos en el bienestar de otras personas. Haidt (2001, 2012) no desconoce que los dos últimos sucesos puedan acontecer, sólo indica que ambos recursos no son frecuentes; la investigación empírica demuestra una primacía de los primeros cuatro fenómenos descritos que acompañan un juicio moral (Greene, 2013; Greene \& Haidt, 2002; Haidt, 2001, 2006, 2007, 2012).

Ahora bien, ¿̇este modelo del juicio moral puede usarse de forma idéntica para explicar la decisión moral? Haidt $(2001,2012)$ no ofrece claridad al respecto. En la explicación del modelo parece suponer que los procesos psicológicos descritos que acompañan el juicio moral son idénticos o acontecen de la misma forma en la decisión y la acción moral. ¿Qué evidencia empírica existe al respecto? ¿La misma evidencia empírica que existe sobre el juicio moral puede extrapolarse para explicar la decisión moral? ¿Con los mismos seis fenómenos o vínculos propuestos en The Social Intuitionist Moral (SIM)? Las críticas a la propuesta explicativa del autor, resaltan la ambigüedad o poca claridad al distinguir el juicio, la decisión y la acción moral (Hershberger, 2014; Jacobson, 2008; Narvaez, 2008; Pizarro \& Bloom, 2003). De tal 
forma, que el modelo de Haidt (2001, 2012) plantea una explicación del juicio moral pero no lo hace con la decisión y la acción moral. Para algunos autores racionalistas, aún el modelo no cuenta con argumentos sólidos que respalden la misma explicación para la decisión moral. $\mathrm{Al}$ parecer, es un artículo aún por escribir en esta propuesta explicativa de la moral. Narváez (2008) considera que la tesis de Haidt (2001, 2012) es útil para explicar el juicio moral pero no es conveniente usar la misma consideración en la decisión moral. Según el autor, el juicio moral y la decisión moral tienen características especiales que exigen explicaciones distintas. Narváez (2008) argumenta que el juicio moral evidentemente es un juicio estético sobre una situación, de tal forma que la intuición tiene una importancia notable como reacción inicial. Sin embargo, la decisión moral es sólo parcialmente estética, es decir, implica, además de una valoración intuitiva también una reflexión evaluativa (consciente y deliberada) sobre las posibles consecuencias que tal decisión pueda tener en el bienestar de otros. En otras palabras, en el juicio moral es suficiente la intuición, pero en la decisión moral, además de la intuición, es inevitable deliberar. Al respecto, Haidt \& Bjorklund (2008b: 242-243) mencionan lo siguiente:

We have focused our writings on moral judgment, but we have never before been explicit that the SIM as published applies only to moral judgment, not to choices about morally relevant actions. We suggest that the SIM can be altered slightly to become a model of moral choice: Just make links 5 (reasoned judgment) and 6 (private reflection) into solid lines and state that "when making real behavioral choices, people often do deliberate." (...) Thus modified, the SIM still retains three advantages over a straight rationalist approach to moral decision making: first, for most morally relevant actions, there is no deliberation; we all do the right thing most of the time without thinking about it. Even heroes who jump into rushing rivers to save people's lives generally state, when interviewed afterwards, that they didn't think about it; they just acted. The importance of automaticity in moral judgment can therefore be brought into moral action as well. Second, when deliberation does occur, it is often biased by desire and an uneven search for evidence. Who would deny that people are extremely good at finding reasons to do what they want to do?. Third, the phenomenology of moral choice blends intuition and conscious deliberation. In Narváez's metaphor for her own experience, "intuition danced with conscious reasoning, taking turns doing the leading" during her attempts at "feeling out' consequences of different decisions. 
En esta respuesta que ofrecen ambos autores a la objeción de Narváez (2008), reconocen que el fenómeno de la decisión moral puede llegar a combinar la intuición con la deliberación consciente. Es decir, que en algunos momentos la intuición y la deliberación pueden funcionar de forma más o menos equilibrada, a diferencia de lo que sucede con el juicio moral. Aunque tampoco renuncian a la idea de que las decisiones o acciones morales también se ejecutan de forma automática la mayoría de las veces. Por un lado, la reflexión privada es útil a la decisión moral en la medida que nos permite tener un diálogo con nosotros mismos y, por otro, la deliberación consciente posibilita ofrecer una respuesta que combina el proceso automático con el deliberado. La razón no solo sirve para justificar o persuadir a otros sobre una decisión moral, sino que también es útil para asumir una distancia reflexiva sobre los motivos psicológicos que configuran aquellas creencias morales que impulsan la ejecución automática de elecciones morales. Estas creencias morales pueden llegar a ser diversas en función del sistema moral concreto que las personas hayan incorporado en su experiencia cultural y psicológica. La deliberación consciente permite contemplar una alternativa distinta a la sugerida por un automatismo moral. Sin embargo, ésta por sí sola no es garantía para generar un cambio en la acción moral y en las intuiciones y emociones que motivan también la ejecución de juicios y decisiones morales. El modelo explicativo propuesto por Haidt $(2001,2012)$ aún requiere un mayor desarrollo teórico, con la consecuente reunión de evidencia empírica, que permita esclarecer la interacción entre la deliberación consciente y la reflexión privada para cuestionar y modificar los procesos automáticos que subyacen a las decisiones morales. No obstante, las ideas del autor representan un punto de partida para comprender la compleja relación por una parte, entre los procesos automáticos y controlados en el comportamiento moral y, por otra, la posible interacción, desde esta concepción dual de la mente, entre el juicio, la decisión y la acción moral. A continuación, se presenta otro modelo explicativo que incorpora las ideas de Haidt (2001, 2012) sobre la cognición moral, con aquello que Gigerenzer (2008a, 2008b) denomina como cognición heurísti- 
ca. Este modelo permite ilustrar con mayor claridad las tesis de Haidt (2001, 2012) aplicadas a la decisión moral, usando como complemento la idea de racionalidad limitada.

\section{Moral y racionalidad limitada}

Un asunto preocupa al ser humano desde la adquisición de la conciencia de sí mismo: la incertidumbre. ¿Podemos concebir alguna estrategia que permita asumir una elección en situaciones desconocidas? Es decir, ¿podemos intentar tener a disposición un recurso o herramienta de elección cuando no es posible conocer con exactitud las variables en juego y las consecuencias en un futuro? Algunos autores concuerdan en resaltar las estrategias culturales que los seres humanos han ideado para resolver la compleja tarea de tomar una decisión. Entre las estrategias más llamativas están: la adivinación (con todas sus variaciones, desde el uso de sortilegios como la lectura de cartas y huesos hasta la observación de hojas de té «taseografía» y el examinar hígados de animales sacrificados «hepatoscopia», pasando por la numerología y la astrología) y los juegos de azar (lanzamiento de monedas y dados, juegos de cartas, entre otros) (Dennett, 2007; Taleb, 2011). Según Dennett (2007), históricamente estas estrategias en la toma de decisiones han sido muy atractivas porque aceleran el proceso de escoger una alternativa, entre varias opciones posibles, como dispositivos que eligen por nosotros. En este caso se ha preferido hacer uso de la fortuna, el destino o el oficio de los dioses para explicar no sólo el curso de los acontecimientos, sino también el resultado y la calidad de las decisiones tomadas. En palabras del autor:

Nos gusta tener razones para hacer lo que hacemos, pero algunas veces nada es suficientemente persuasivo nos viene a la mente, y como reconocemos que debemos decidir pronto, nos ingeniamos un pequeño dispositivo, un objeto externo que tomará las decisiones por nosotros. Pero si la decisión atañe a algo de gran trascendencia, como si ir o no a la guerra, o casarse, o confesar, cualquier cosa que se le parezca a arrojar una moneda al aire será, digamos, bastante frívola. En tal caso, hacer una elección sin tener una buena razón sería un signo de incompetencia demasiado obvio; además, si la decisión es realmente tan impor- 
tante, una vez que la moneda ha caído al suelo hay que enfrentarse a una elección adicional: ¿̇tendré que cumplir con el compromiso que acabo de aceptar y acatar lo que diga la moneda, o será mejor que lo reconsidere? Enfrentados a estos dilemas, reconocemos la necesidad de algún tratamiento más fuerte que el de arrojar una moneda. Algo más ceremonial, más impresionante, como la adivinación, que no sólo nos dice qué hacer sino que, además, nos da una razón (si aguzamos la vista lo suficiente y usamos la imaginación) (Dennett, 2007, p.167).

Las estrategias han sido simples o complejas en función de la transcendencia o no de las circunstancias que demanden una elección. Inventamos trucos, buscamos ayuda, delegamos las circunstancias al deseo de seres sobrenaturales o al destino. A pesar de estas maniobras que aún viven y se transforman en algunos contextos culturales, la matemática francesa del siglo Xvir y xviI asumió el reto de hallar una pauta de comportamiento óptima para tomar una decisión en condiciones azarosas (León, 1987). Y esto, de acuerdo con León (1987), a través de la consecución de dos propósitos: "describir de la mejor forma posible la conducta del sujeto racional (que sería el objetivo más psicológico, por decirlo así) y por otro lado (...) deducir cuál es la mejor forma de decidir, suponiendo que se quiere obtener el máximo de beneficio" (p.82). La ciencia moderna de la decisión se articuló rápidamente al intento de medir la conducta en la toma decisiones y al mismo tiempo descubrir, con la matemática y la estadística como herramientas, la mejor opción posible en diversos contextos de elección. Sin embargo, un supuesto problemático subyacía a la teoría económica moderna: los seres humanos son racionales y, por lo tanto, buscan siempre en sus decisiones el mayor beneficio posible para sí mismos, evaluando todas las opciones de elección (Pena, 2005). Tal supuesto generó una tensión contemporánea entre los modelos descriptivos (derivados especialmente de la psicología) que han intentado describir y explicar cómo los individuos toman decisiones, y los modelos normativos (surgidos de la matemática y la teoría económica neoclásica), que suponen cómo se debería elegir, apelando al uso exclusivo de la razón para evaluar con detenimiento todas las opciones de respuesta y escoger la mejor alternativa en beneficio propio (León, 1987 Kahneman, 2012; Pena, 2005). Disponer de una herramienta matemática para decidir, sope- 
sando numéricamente las opciones de respuesta, supone disponer de una estrategia confiable que permita concebir con precisión la mejor opción a elegir.

La propuesta de Gigerenzer (2008a, 2008b, 2000c) surge en oposición a este modelo clásico de racionalidad. Según el autor, la racionalidad humana es limitada, ésta utiliza trucos o atajos para tomar decisiones obteniendo con frecuencia resultados convenientes. Esta tendencia mental de usar atajos en la elección sin evaluar con detenimiento todas las alternativas, es denominada por Gigerenzer (2000a, 200ob) "cognición heurística”, una cognición basada en atajos que ha evolucionado en un mundo envuelto por la niebla de la incertidumbre. Ante el esfuerzo, la energía y el tiempo que demanda evaluar todas las alternativas (sugerencia de racionalidad presente en la teoría económica), usamos la intuición para escoger una opción llamativa sin evaluar con precisión todas las opciones. En este escenario, según el autor, emprendemos "un viaje a un casi desconocido territorio de racionalidad, poblado por personas como nosotros, parcialmente ignorantes, con el tiempo limitado y el futuro incierto" (Gigerenzer, 2008b, 10). En estas condiciones, ignorantes, sin tiempo disponible y un futuro desconocido, la intuición adquiere una importancia notable.

Un ejemplo llamativo del autor en relación con la elección de pareja entre dos alternativas (A o B) y la recomendación racionalista de evaluar teniendo en cuenta, con una lista, todas las razones posibles a favor o en contra de cada alternativa:

Harry se sintió muy aliviado al saber que existía una fórmula lógica para resolver su problema. Así que se tomó su tiempo, anotó todas las razones importantes que se le ocurrieron, las sopesó con cuidado y pasó al cálculo. Al ver el resultado, sucedió algo inesperado. Una voz interior le decía que aquello no estaba bien. Y por primera vez, Harry se dio cuenta de que corazón ya había decidido, contra el cálculo y a favor de la otra chica. La operación ayudaba a encontrar la solución, pero no gracias a su lógica. Basándose en razones para él desconocidas, llevó una decisión inconsciente a su consciencia (Gigerenzer, 2008b, p.11). 
El autor utiliza de forma intercambiable las siguientes expresiones para referirse a lo mismo: reacciones instintivas, sentimientos viscerales, intuiciones o corazonadas. Una intuición alude a una evaluación que: “1. Aparece rápidamente en la consciencia, 2. De cuyas razones subyacentes no somos plenamente conscientes; y, 3. Que es lo bastante fuerte para que actuemos en función de la misma" (Gigerenzer, 2008b: 23). Asimismo, la intuición tiene dos componentes: "1. Simples reglas generales, que sacan provecho de: 2. Facultades evolucionadas del cerebro" (Gigerenzer 2008b, 25). Las reglas generales que fundamentan la intuición son atajos (heurísticos) que utilizan la información más importante para tomar decisiones, ignorando otros datos o fuentes de información. Estas reglas se articulan, por ejemplo, al primer borrador moral ofrecido por la naturaleza (facultades evolucionadas del cerebro como las que mencionan Haidt (2012) y Haidt \& Joseph (2008), a propósito de las seis papilas gustativas de la moral; estos rasgos universales de por sí son reglas generales que motivan intuiciones) y son transformadas por la práctica prolongada durante toda la vida con la cultura. Estas ideas riñen o se oponen al dogma racionalista de la toma de decisiones, según el cual los seres humanos tienen una racionalidad que les permite elegir la mejor acción posible después de evaluar de forma deliberada todas las opciones de respuesta. Al contrario, la racionalidad es limitada y normalmente tomamos decisiones guiados por la intuición sin necesidad de considerar todas las posibilidades de acción o sin razonar de forma permanente. Además del recurso natural y limitado que la especie humana tiene en relación con la reflexión consciente, según Gigerenzer (2008b, 26): "podemos considerar que la mente es una caja de herramientas adaptativa con reglas creadas y transmitidas genética, cultural e individualmente". Y la misma concepción aplica para explicar las intuiciones propias de la moral. Es decir, la moral es un rasgo biológico y cultural de la mente como una caja de herramientas adaptativa. Podemos usar estas "reglas creadas y transmitidas genética, cultural e individualmente" para tomar decisiones económicas, políticas o morales. En este modelo explicativo, el autor no presta atención al proceso del razonamiento consciente ni a la res- 
puesta deliberada que se apoya en él, sino que se ocupa de forma exclusiva en explicar el proceso de la intuición y en la respuesta automática basada en el mismo con aplicaciones diversas en distintos contextos de elección (Gigerenzer, 2008a, 2008b, 2008c, 2008d, 2010; Gigerenzer \& Sturn, 2012; Kruglansk \& Gigerenzer, 2011).

Es importante señalar la distinción que hace Gigerenzer (2008b) entre maximizadores y satisfactores. Los individuos maximizadores son aquellos que buscan la mejor alternativa para sí mismos (el agente racional propuesto por la teoría económica), la opción de respuesta que maximice su placer inmediato. De acuerdo con el autor, estas personas sólo existen en la ficción de la teoría económica, en la medida en que, si las personas actuaran así, nunca lograrían cumplir su cometido; debido a la incertidumbre no hay garantías de elegir la mejor alternativa posible. En cambio, los individuos satisfactores son aquellos que tienen una racionalidad limitada, guiada fundamentalmente por intuiciones, que adoptan "rápidamente la primera alternativa satisfactoria o razonablemente buena" (Gigerenzer, 2008b, 13). El autor no desconoce que hay individuos que intentan ser maximizadores, pero la cuestión es que estos nunca logran garantizar y demostrar una maximización en sus decisiones; además, es más frecuente encontrar en la vida cotidiana individuos satisfactores. Aquellas personas que procuran ser maximizadoras, en busca siempre de lo mejor, se ven acosadas habitualmente por sentimientos depresivos o melancólicos y emociones de perfección, arrepentimiento y autocensura (Gigerenzer, 2008a, 2008b; Kahneman, 2012; Taleb, 2011; Tiberius, 2015).

De acuerdo con Gigerenzer (2008b, 2008c, 2010), el juicio, la decisión y la conducta moral es satisfactoria, no es en ningún modo maximizadora. Es decir, cuando asumimos un juicio de aprobación o censura moral o tomamos una decisión moral, lo hacemos por intuición apoyados en la primera alternativa u opción de respuesta satisfactoria, razonablemente buena. Gigerenzer (2008b, 206-207) propone los siguientes tres principios que explican las intuiciones morales (aquellas que subyacen a toda decisión moral como respuesta automática): 
No ser consciente. Una intuición moral, como otras reacciones instintivas, aparece rápidamente en la consciencia, es lo bastante fuerte para que actuemos con arreglo a la misma y no es posible verbalizar su lógica subyacente.

Raíces y reglas. La intuición está vinculada a una de tres "raíces" (individual, familiar ampliada o comunitaria) y a un objetivo emocional (por ejemplo, evi$\operatorname{tar}$ daño), y puede describirse mediante reglas generales. No son forzosamente propias de la conducta moral, pero subyacen a otras acciones.

Entorno social. La conducta moral está supeditada al entorno social. Algunos desastres morales pueden evitarse si uno conoce las reglas que orientan la conducta de las personas y los entornos que desencadenan estas reglas.

El primer principio hace referencia a las intuiciones morales que se experimentan como ocurrencia, presentimiento o sentimiento moral. El segundo principio implica la existencia de tres raíces morales distintas: la ética individualista, familiar o comunitaria. La ética individualista privilegia los derechos y libertades individuales, una ética propia de la convicción liberal en las sociedades occidentales. La moral familiar, enfatiza en una obligación moral de por vida con la familia. En este caso, los sentimientos morales se relacionan con la familia más que con el individuo. Y la ética comunitaria incluye obligaciones morales en función de la lealtad al grupo y el respeto a la autoridad grupal. Cada contexto cultural enfatiza una u otra raíz. Por ejemplo, si un individuo, además de pertenecer a una sociedad que privilegia una ética individualista, ha desarrollado ésta en su cognición moral, las intuiciones que experimentará este individuo en sus decisiones morales con frecuencia van a privilegiar el derecho y la libertad individual. De igual forma, acontece con las otras dos raíces morales: familiar y comunitaria (más frecuente en una convicción política conservadora o en alguna creencia religiosa) (Gigerenzer, 2008b, 2008c, 2010). Finalmente, el tercer principio alude a los entornos sociales que fundamentan ciertos tipos de conducta moral. En la medida en que estos entornos sociales sean diversos, es muy probable que lo mismo acontezca con la conducta moral. Los entornos sociales, además de ser diversos, pueden llegar a interactuar en un contexto espacio-temporal próximo. Por ejemplo, el acceso a la información virtual, con internet y redes sociales, posibi- 
lita estas interacciones entre sistemas morales diversos que corresponden a contextos sociales distintos.

Gigerenzer (2008b, 2010) relaciona estos principios con las cinco capacidades evolutivas (papilas gustativas) de la moral propuestos por Jonathan Haidt (2012): cuidado/daño; justicia/reciprocidad; lealtad al grupo; autoridad/respeto; y pureza/santidad. En síntesis: daño, reciprocidad, jerarquía, grupo excluyente y pureza. Por ejemplo, según Gigerenzer (2008b), una sociedad y un individuo con una ética individualista enfatiza y desarrolla con mayor grado e intensidad las dos primeras papilas gustativas (el daño y la reciprocidad). En tal caso, las intuiciones morales que se experimentan con mayor frecuencia están orientadas a preservar la autonomía personal en temas como el derecho al aborto, la eutanasia, la libre expresión, la identidad sexual, el libre consumo de marihuana, entre otros. Una sociedad y un individuo con una ética familiar activan, de igual forma, las dos primeras capacidades evolutivas (daño y reciprocidad), pero en tal caso los sentimientos morales de daño y reciprocidad se relacionan más con la familia que con el individuo. Los temas de interés moral aquí son el bienestar y el honor de la familia. Y finalmente, una sociedad o individuo con una ética comunitaria con frecuencia activan las cinco capacidades evolutivas. En tal caso las intuiciones morales privilegian la comunidad teniendo en cuenta todas las papilas gustativas de la moral. En palabras de Gigerenzer (2008b, 209):

La mayoría de las tribus, los grupos religiosos o las naciones defienden virtudes de patriotismo, lealtad, heroísmo, y desde tiempo inmemorial los individuos han sacrificado la vida por su grupo excluyente. En época de guerra, el "apoyo a nuestras tropas" es el sentimiento patriótico predominante, y criticarlo se considera traición. Del mismo modo, la mayoría de comunidades tienen un código de pureza, contaminación y divinidad. Las personas se sienten indignadas cuando se infringe este código (...) Mientras que en los países occidentales las cuestiones morales suelen centrarse en la libertad personal (como el derecho a poner fin a la vida de uno), en otras sociedades la conducta moral se centra más en la ética de la comunidad, incluyendo aquí el deber, el respeto y la obediencia a la autoridad. 
Desde esta perspectiva, la especie humana posee una predisposición a la moral con tres raíces culturales (individualista, familiar y comunitaria) y cinco capacidades evolutivas distintas (daño, reciprocidad, jerarquía, grupo excluyente y pureza). La combinación de estas disposiciones naturales y culturales genera sistemas morales distintos que motivan, por ejemplo, en situaciones de elección idénticas, decisiones morales distintas. Por ejemplo, si un individuo cuenta con la posibilidad de votar a favor o en contra de una ley o política que permita el aborto, es posible que vote a favor guiado por una intuición moral que se deriva de una raíz moral individualista (y la capacidad de cuidado/daño y reciprocidad) que en consecuencia privilegia la autonomía personal. Sin embargo, otra persona, en un contexto de elección moral idéntico, es posible que vote en contra de la autorización del aborto. $\mathrm{Y}$ esto motivado por una ética comunitaria que protege los intereses y creencias de algún grupo religioso, con énfasis en la capacidad evolutiva del grupo excluyente y la santidad/pureza. En este último caso, alguna prescripción religiosa puede considerar el aborto como la violación de un mandamiento divino: "no matarás". Resulta entonces inaceptable ceder a la posibilidad de "asesinar una vida" en proyecto de concepción y maduración orgánica en el vientre de la madre. En definitiva, para Gigerenzer (2008b, 228) "las intuiciones morales se basan en reglas generales que se apoyan en la toma de decisiones a partir de una sola razón más que en sopesar y sumar consecuencias”. No obstante, ¿podemos confiar por defecto en nuestras intuiciones morales?¿Cómo identificar y resistir aquellas intuiciones morales que pueden generar, a través de la toma de decisiones, consecuencias desafortunadas? Si hay una primacía de la intuición en la decisión moral, ¿̇cómo diseñar y ofrecer una formación en la sociedad contemporánea que permita a infantes, adolescentes y adultos tomar decisiones morales responsables? ¿Qué relación tiene la responsabilidad moral con los sentimientos morales? ¿En qué medida somos responsables cuando nuestras decisiones morales son motivadas con frecuencia por intuiciones morales? (Driver \& Loeb, 2008; Gigerenzer, 2008d; Haidt \& Bjorklund, 2008b; Pizarro \& Bloom, 2003; Sadler-Smith, 2008; Suhler \& Churchland, 2011; Suns- 
tein, 2005, 2008). Estas inquietudes resaltan las objeciones más relevantes al modelo sentimentalista de Haidt $(2001,2012)$ y de Gigerenzer (2008b, 2008c, 2010). En el siguiente apartado, se considera una explicación de la decisión moral como respuesta automática con las ideas presentes en los modelos naturalistas propuestos por ambos autores.

\section{Decisión moral. Entre los modelos explicativos}

La especie humana tiene la competencia natural de tomar decisiones morales de forma automática o deliberada. En ambos casos, como rutas complementarias de elección, la intuición y la emoción moral constituyen un recurso para considerar alguna opción preferible sobre las demás. En otras palabras, cuando una decisión moral se toma de forma automática, con la intuición y la emoción es suficiente para optar por una alternativa. Por otro lado, cuando una decisión moral se toma de forma deliberada interviene la reflexión y evaluación de las opciones en juego pero con la presencia de una señal intuitiva y emocional que ofrece una pista de elección. En la propuesta explicativa de Haidt (2001, 2006, 2012), en cualquier situación que demande un juicio o una decisión la respuesta inmediata es automática y luego usamos el razonamiento con el propósito de justificar y persuadir; sólo en contadas ocasiones el ser humano utiliza la reflexión privada y la deliberación consciente, en el ámbito moral, para considerar y elegir alternativas distintas a la sugerida por una respuesta automática. Haidt (2001, 2006, 2012) sugiere que estas últimas dos capacidades pueden ejecutarse pero esto no acontece con frecuencia, esta idea se infiere de la evidencia empírica presentada por el autor. En otras palabras, en el juicio y la decisión moral, la deliberación consciente adquiere un papel menor. Además, las respuestas automáticas están arraigadas a sistemas morales que se comparten entre grupos de personas y que son incorporados en el transcurso de la vida. Por tal razón, la dificultad que representa para un ser humano modificar o cambiar una intuición moral, en tanto ésta se encuentra arraigada en una creencia, una norma o un valor compartido (Haidt \& Kesebir, 2008). También, es 
importante resaltar que las ideas de Haidt (2001, 2006, 2012) invitan a pensar en la interacción compleja y sesgada de los procesos automáticos a los procesos controlados en el comportamiento moral pero, de igual forma, desde el modelo explicativo no es posible determinar con precisión en las decisiones morales en qué medida el ser humano utiliza su capacidad para deliberar, como una respuesta rebelde frente al automatismo moral, y qué circunstancias culturales motivan este uso de la deliberación consciente.

Por su parte, Gigerenzer (2008b, 2010) retoma las ideas de Haidt (2012) sobre las capacidades evolucionadas del comportamiento moral, que posibilitan en la especie el despliegue de intuiciones y emociones, y, además, introduce el concepto de satisfacción en la racionalidad limitada. En este sentido, los seres humanos cuentan con la competencia de elegir aquellas alternativas que resulten lo suficientemente buenas sin apelar a la necesidad de escoger la mejor opción posible. Esta posibilidad surge de una racionalidad limitada por todas las restricciones que debe enfrentar la persona en su toma de decisiones cotidianas. En palabras de Churchland (2012, 35-37):

\begin{abstract}
Por norma general, la toma de decisiones es un asunto de restricciones y satisfacciones, y cuando se desarrolla bien, podemos afirmar que ha prevalecido la racionalidad. Pero aún más complejo que la toma de decisiones es el asunto de abordar problemas sociales dentro de un grupo social, en el que los intereses en liza, las creencias, los temperamentos y las tradiciones restringen la decisión que establece el grupo, y donde cada cerebro individual aporta su propio conjunto de restricciones internas (...) A menudo se barajan opciones mejores o peores, pero no existe una única opción correcta; en estos casos, la pauta de satisfacción por restricción realiza su tarea, que consiste en equilibrar, armonizar y, por último, establecer una decisióna adecuada.
\end{abstract}

En este ejercicio permanente de obtener satisfacción con restricción en las decisiones morales, Gigerenzer (2008b, 2010) plantea la importancia que tiene la intuición moral como una brújula de elección en medio de todas las alternativas y restricciones que enfrenta el ser humano en su vida cotidiana. Por esta razón, los automatismos morales, 
adquiridos por aprendizaje consciente o inconsciente (Barg \& Chartrand, 1999; Churchland, 2012; Churchland \& Suhler, 2014; Damasio, 2010; Wilson, 2002), representan un truco de navegación en la incertidumbre porque nos libran también de tener que acudir a la deliberación consciente de forma permanente. En síntesis, Haidt $(2006,2012)$ y Gigerenzer $(2008 b, 2010)$ resaltan la importancia del automatismo en la decisión moral, con los respectivos matices teóricos que adquieren cada una de sus propuestas explicativas. Ambos autores sugieren que el depliegue de la intuición y la emoción moral constituyen herramientas evolutivas, más antiguas que la deliberación consciente, que aun inciden en la ejecución de nuestras decisiones morales.

\section{Conclusión}

Las propuestas de Haidt y Gigerenzer resaltan el papel de la intuición y la emoción en las decisiones morales. De tal forma que estas respuestas automáticas son ubicuas en las decisiones cotidianas, constituyen un recurso para enfrentar la incertidumbre y sesgan en la deliberación para tomar una decisión moral. Sin embargo, ambos autores restan importancia, en sus propuestas explicativas, al uso que puede tener la deliberación para cuestionar los automatismos ya establecidos e incorporar nuevos automatismos en la decisión moral. Las respuestas automáticas se organizan y despliegan con base al aprendizaje individual y al sistema moral incorporado en el comportamiento, este sistema se compone de creencias morales compartidas entre un grupo de seres humanos. De esta forma, en toda intuición y emoción moral subyace una creencia que es adquirida por aprendizaje consciente o inconsciente. En la teoría propuesta por Haidt (2001, 2006, 2012) por defecto tenemos en principio una intuición o emoción, elegimos una alternativa en un problema de elección moral y luego justificamos y persuadimos a otras personas de la decisión tomada. La reflexión privada y la deliberación consciente en contadas ocasiones se utilizan para emitir juicios y tomar decisiones morales. Y en la propuesta teórica de Gigerenzer (2008b, 2010) la intuición y emoción moral constituye 
un recurso para elegir en condiciones de incertidumbre, de ahí que la opción elegida sea satisfaciente, es decir, una alternativa que es razonablemente buena para el individuo sin tener la exigencia de escoger la mejor opción en una decisión moral. En ambas propuestas teóricas es necesario precisar la interacción de la deliberación consciente con estos automatismos que en ocasiones representan recursos oportunos para decidir pero que en otros casos estos pueden llegar a ser inadecuados. La preferencia que exalta una intuición o emoción moral no supone por defecto que la opción de interés siempre sea la más adecuada, sin embargo, estos recursos automáticos ofrecen a la especie humana una señal afectiva, una pista de elección moral en la incertidumbre.

\section{Referencias}

Antony, L. (2000). Naturalized Epistemology, Morality, and the Real World. In: Campbell, R., and Hunter, B. (Eds.), Moral Epistemology Naturalized (pp.103138). The Canadian Journal of Philosophy. University of Calgary Press.

Babbitt, S. (2000). Moral Naturalism and the Normative Question. In: Campbell, R., and Hunter, B. (Eds.), Moral Epistemology Naturalized (pp.139-174). The Canadian Journal of Philosophy. University of Calgary Press.

Barg, J., \& Chartrand, T. (1999). The Unbearable Automaticity of Being. American Psychologist, 54(7), 462-479. Recuperado de http://dx.doi.org/10.1037/0003-066X.54.7.462

Bartels, D. (2008). Principled moral sentiment and the flexibility of moral judgment and decision making. Cognition, 108(2), 381-417. doi:10.1016/j.cognition.2008.03.001

Bartels, D., Bauman, C., Cushman, F., Pizarro, D., and McGraw, P. (2015). Moral Judgment and Decision Making. In Keren, G., and Wu, G. (Eds.), The Wiley Blackwell Handbook of Judgment and Decision Making (pp.1-51). Chichester, UK: Wiley.

Camps, V. (2013). Breve historia de la ética. Barcelona: RBA Libros S.A. 
Cela, C. (1999). Del naturalismo contemporáneo. De Darwin a la sociobiología. En: Camps, V. (Ed.), Historia de la ética. III. La ética contemporánea (pp.601634). Barcelona: Crítica.

Chang, R. (2009). Voluntarist reasons and the sources of normativity. In: Sobel, D., and Wall, S. (Eds.), Reasons for action (pp.243-271). Cambridge: Cambridge University Press.

Chang, R. (2012). Are hard choices cases of incomparability? Philosophical Issues. Action Theory, (22), 106-126. Recuperado de http://ruthchang.net/wp-content/uploads/2012/11/PAre-Hard-Choices-cases-of-incomparability.pdf.

Churchland, Patricia S. (2006). Moral decision-making and the brain. In: Illes, J. (Ed.), Neuroethics. Definning the issues in theory, practice and policy (pp.316). Oxford: Oxford University Press.

Churchland, Patricia S., \& Suhler, C. (2014). Agency and Control: The Subcortical Role in Good Decisions. In: Sinnott-Armstrong, W. (Ed.), Moral Psychology. Free Will and Moral Responsibility. 4, (pp.309-326). MIT Press: Massachusetts Institute of Technology.

Churchland, P. (2012). El cerebro moral. Lo que la neurociencia nos cuenta sobre la moralidad. Barcelona: Paidós.

Damasio, A. (1999). El error de Descartes. La razón de las emociones. Barcelona: Editorial Andres Bello.

Damasio, A. (2007). En busca de Spinoza. Neurobiología de la emoción y los sentimientos. Barcelona: Crítica.

Damasio, D. (2010). Y el cerebro creó al hombre. ¿Cómo pudo el cerebro generar emociones, sentimientos, ideas y el yo? Barcelona: Ediciones Destino, S.A.

Dennett, D. (2004). La evolución de la libertad. Barcelona: Ediciones Paidós Ibérica, S.A.

Dennett, D. (2007). Romper el hechizo. La religión como fenómeno natural. Barcelona: Katz Editores.

De Waal, F. (2007). Primates y filósofos. La evolución de la moral del simio al hombre. Barcelona: Ediciones Paidós Ibérica. 
Driver, J., Loeb, D. (2008). Moral heuristics and consequentialism. In: Sinnott-Armstrong, W. (Ed.), Moral Psychology. 2. The Cognitive Science of Morality: Intuition and Diversity (pp.31-40). Cambridge: The MIT Press.

Gigerenzer, G. (2008a). Rational for Mortals. How People Cope with Uncertainty. Oxford: Oxford University Press.

Gigerenzer, G. (2008b). Decisiones instintivas. La inteligencia del inconsciente. New York: Penguin Group.

Gigerenzer, G. (2008c). Moral intuition. Fast and frugal heuristics? In: Sinnott-Armstrong, W. (Ed.), Moral Psychology. 2. The Cognitive Science of Morality: Intuition and Diversity (pp.1-26). Cambridge: The MIT Press.

Gigerenzer, G. (2008d). Reply to comments. In: Sinnott-Armstrong, W. (Ed.), Moral Psychology. 2. The Cognitive Science of Morality: Intuition and Diversity (pp.41-46). Cambridge: The MIT Press.

Gigerenzer, G. (2010). Moral Satisficing: Rethinking Moral Behavior as Bounded Rationality. Topics in Cognitive Science, 2(3), 528-554. doi: 10.1111/j.17568765.2010.01094.x.

Gigerenzer, G., \& Sturn, T. (2012). How (far) can rationality be naturalized? Synthese, 187(1), 243-268. doi 10.1007/s11229-011-0030-6.

Greene, J. (2013). Moral Tribes. Emotion, Reason and The Gap Between Us and Them. New York: The Penguin Press.

Greene, J., \& Haidt, J. (2002). How (and where) does moral judgment work? Trends in Cognitive Sciences, 6(12), 517-523. doi: http://dx.doi.org/10.1016/S13646613(02)02011-9.

Haidt, J. (2001). The emotional dog and its rational tail: A social intuitionist approach to moral judment. Psychological Review, 108(4), 814-834. doi: 10.1037//0033-295X. 108.4.814.

Haidt, J. (2006). La hipótesis de la felicidad. La búsqueda de verdades modernas en la sabiduría antigua. Barcelona: Editorial Gedisa, S.A. 
Haidt, J. (2007). The new synthesis in moral psichology. Science, 316, 998-1002. doi: 10.1126/science.1137651.

Haidt, J. (2012). The righteous mind. Why good people are divides by politics and religion. New York: Vintage Books.

Haidt, J., \& Joseph, C. (2008). The moral mind: How five sets of innate intuitions guide the development of many culture-specific virtues, and perhaps even modules. In: Carruthers, P., Laurence, S., Stich, S., (Eds.), The innate mind. Foundations and the future. Evolution and cognition, 3 (pp.367-391). New York: Oxford University Press.

Haidt, J., \& Bjorklund, F. (2008a). Social Intuitionists Answer Six Questions about Moral Psychology. In: Sinnott-Armstrong, W. (Ed.), Moral Psychology, 2. The Cognitive Science of Morality: Intuition and Diversity (pp.181-218). Cambridge: The MIT Press.

Haidt, J., \& Bjorklund, F. (2008b). Social Intuitionists Reason, in Conversation. In: Sinnott-Armstrong, W. (Ed.), Moral Psychology, 2. The Cognitive Science of Morality: Intuition and Diversity (pp.241-254). Cambridge: The MIT Press.

Haidt, J., \& Kesebir, S. (2008). In the Forest of Value: Why Moral Intuitions Are Different from Other Kinds. In: Plessner, H., Betsch, C., \& Betsch, T. (Eds.), Intuition in Judment and Decision Making (pp.209-230). New York: Taylor \& Francis Group.

Hershberger, J. (2014). Reason, Society, and the Social Intuitionist Model. Compos mentis: Undergraduate Journal of Cognition and Neuroethics, 2(2), 29-43. Recuperado de http://www.cognethic.org/cm/cmv2i2_Hershberger.pdf.

Hume, D. (2014). Investigación sobre los principios de la moral. Prólogo, traducción y notas de Carlos Mellizo. An Inquiring Concerning The Principles of Morals. 1751. Alianza Editorial.

Illiev, R., Sachdeva, S., Bartels, D., Joseph, C., Suzuki, S., and Medin, D. (2009). Attending to Moral Values. In Bartels, D., Bauman, C., Skitka, L., \& Medin, D. 
(Eds.), Moral Judgment and Decision Making (pp.169-192). Oxford: Elsevier's Science \& Technology Rights Department.

Jacobson, D. (2008). Does social intuitionism flatter morality or challenge it? In:

Sinnott-Armstrong, W. (Ed.), Moral Psychology, 2. The Cognitive Science of Morality: Intuition and Diversity (pp.219-232). Cambridge: The MIT Press.

Kahneman, D. (2012). Pensar rápido, pensar despacio. Barcelona: Random House Mondadori, S.A.

Koorsgard, C. (2004). Actuar por una razón. Anuario Fỉlosófico, хххVII(3), (645-677).

Recuperado de http://dadun.unav.edu/bitstream/10171/4577/1/KORSGAARD.pdf.

Koorsgard, C. (2007). La moralidad y la singularidad de la condición humana. De Waal, F. (Ed.), Primates y filósofos. La evolución de la moral del simio al hombre (pp.131-154). Barcelona: Ediciones Paidós Ibérica.

Kruglanski, A., \& Gigerenzer, G. (2011). Intuitive and Deliberate Judgments Are Based on Common Principles. Psychological Review, 118(1), (97-109).

León, O. (1987). La toma de decisiones individuales con riesgo desde la psicología. Infancia y Aprendizaje, 30, (81-94). Recuperado de https://dialnet.unirioja. es/servlet/articulo?codigo $=65980$.

Narvaez, D. (2008). The Social-Intuitionist Model: Some Counter-Intuitions. In Sinnott-Armstrong, W. (Ed.), Moral Psychology, 2. The Cognitive science of morality: Intuition and diversity (pp.233-240). Cambridge: The MIT Press.

Nussbaum, M. (1995). El discernimiento de la percepción: una concepción aristotélica de la racionalidad privada y pública. Estudios de Filosofía (Instituto de Filosofía de la Universidad de Antioquia), 11, 107-167. Recuperado de http:// philpapers.org/rec/NUSEDD-2.

Nussbaum, M. (2008). Paisajes del pensamiento. La inteligencia de las emociones.

Barcelona: Ediciones Paidós Ibérica, S.A.

Pena, J. (2005). El problema de la racionalidad en economía o las inconsistencias del Homo oeconomicus. Estudios Filosóficos (Valladolid), 155(54), (33-58). Recu- 
perado de https://dialnet.unirioja.es/servlet/articulo?codigo $=1112590$.

Pizarro, D., \& Bloom, P. (2003). The Intelligence of the Moral Intuitions: Comment on Haidt (2001). Psychological Review, 110(1), (193-196). Recuperado de http://dx.doi.org/10.1037/0033-295X.110.1.193.

Prinz, J. (2007). The Emotional Construction of Morals. Oxford: Oxford University Press.

Prinz, J., \& Nichols, S. (2010). Moral Emotions. In: Doris J., and The Moral Psychology of Moral Group (Eds.), The Moral Psychology Handbook (pp.111-146). Oxford: Oxford University Press.

Sadler-Smith, E. (2008). Inside Intuition. London: Routledge Taylor \& Francis Group.

Suhler, C., \& Churchland, P. (2011). Can Innate, Modular "Foundations" Explain Morality? Challenges for Haidt's Moral Foundations Theory. Journal of Cognitive Neuroscience, 239, (2103-2116). doi: 10.1162/jocn.2011.21637.

Sunstein, C. (2005). Moral Heuristics. Behavioral and Brain Sciences, 28(4), (531573). Recuperado de http://www.ncbi.nlm.nih.gov/pubmed/16209802.

Sunstein, C. (2008). Fast, frugal, and (sometimes) wrong. In: Sinnott-Armstrong, W. (Ed.), Moral Psychology, 2. The Cognitive Science of Morality: Intuition and Diversity (pp.27-30). Cambridge: The MIT Press.

Smith, A. (2012). Teoría de los sentimientos morales. Selección y traducción de Raúl Meléndez Acuña. Medellín: Editorial Universidad de Antioquia.

Taleb, N. (2011). El Cisne Negro. El impacto de lo altamente improbable. Barcelona: Paidós Trancisiones.

Tiberius, V. (2015). Moral Psychology. A Contemporary Introduction. New York: Routledge. Taylor \& Francis Group.

Tomasello, M. (2014). A natural history of human thinking. Cambridge: Harvard University Press.

Turiel, E. (2006). Thought, emotions, and social interactional procesess in moral development. In: Killen, M., and Smetana, J. (Eds.), Handbook of moral deve- 
lopment (pp.7-36). New Jersey: Lawrence Erlbaum Associates, Inc.

Wilson, T. (2002). Strangers to ourselves. Discovering the adaptive unconscious.

Cambridge: Harvard University Press. 